\title{
CDK-associated Cullin 1 can promote cell proliferation and inhibit cisplatin-induced apoptosis in the AGS gastric cancer cell line
}

Qi Zheng ${ }^{1,2}$, Ling-Yu Zhao ${ }^{3}$, Ying Kong ${ }^{1}$, Ke-Jun Nan $^{1 *}$, Yu Yao ${ }^{1}$ and Zi-Jun Liao ${ }^{2}$

\begin{abstract}
Background: Gastric cancer is a common and highly lethal malignancy in the world, but its pathogenesis remains elusive. In this study, we focus on the biological functions of CDK-associated Cullin1 (CAC1), a novel gene of the cullin family, in gastric cancer, which may help us to further understand the origin of this malignancy.

Methods: The AGS and MGC803 gastric cancer cell lines and the GES-1 gastric mucosa cell line were selected for study. At first, $C A C 1$ expressions of those cell lines were examined by quantitative real-time reverse transcription polymerase chain reaction (qRT-PCR) and western blot examinations, then CAC1 small interfering RNA (CAC1-siRNA) were designed and transfected into the AGS cell line with a relatively high level of CAC1. Once CAC1 was silenced, a series of biological characteristics of AGS cells such as cell proliferation, cell cycle, apoptosis, and expressions of apoptosis-related genes (P53, BCL2 and BAX) were determined by MTT, flow cytometry, QRT-PCR and western blot, respectively.

Results: CAC1 expression of AGS or MGC803 was much higher than that of GES-1. After CAC1 expression was effectively depressed by RNA interference in AGS cells, significant cell growth inhibition occurred. Furthermore, the proportion of cells treated with CAC1-siRNA increased in the G1 phase and decreased in the S phase, indicative of G1 cell cycle arrest. More importantly, the proportions of early/late apoptosis in AGS cells were enhanced with cis-diaminedichloroplatinum (cisplatin, CDDP) treatment, but to a higher extent with cisplatin plus CAC1-siRNA. Interestingly, BCL2 mRNA copies showed about a 30\% decrease in the cisplatin group, but dropped by around 60\% in the cisplatin plus CAC1-siRNA group. Conversely, the P53 mRNA expressions obtained nearly a two-fold increase in the cisplatin group, in addition to a five-fold increase in the cisplatin plus CAC1-siRNA group, and the BAX mRNA levels had almost a two- and four-fold augmentation, respectively. Meanwhile, P53, BAX and BCL2 showed the same alteration patterns in western blot examinations.
\end{abstract}

Conclusions: CAC1 can promote cell proliferation in the AGS gastric cancer cell line. Moreover, it can prevent AGS cells from experiencing cisplatin-induced apoptosis via modulating expressions of P53, BCL2 and BAX.

Keywords: Gastric cancer, CDK-associated Cullin1, Proliferation, Cell cycle, Apoptosis

\section{Background}

Gastric cancer is one of the most common malignant tumors and the second leading cause of cancer death in the world, responsible for a total of 989,600 new cases and 738,000 deaths annually [1]. Over past years, there has been a steady decline in the incidence and mortality risk of gastric cancer in most countries [2], due to the tremendous

\footnotetext{
*Correspondence: Nankji@163.com

'Department of Medical Oncology, First-Affiliated Hospital, Xi'an Jiaotong University, 277 Yanta West Road, Xi'an 710061, Shaanxi Province, China Full list of author information is available at the end of the article
}

developments in diagnosis and treatment methods. However, gastric cancer remains a great threat to people, especially those in developing countries [1], and the survival of all affected patients, even after curative surgical resection and adjuvant therapy, is less than $40 \%$ [3].

Epidemiologic investigations have uncovered many risk factors for gastric cancer, and molecular biology research further indicates that gastric carcinogenesis comprises numerous genetic and epigenetic events, involving a cluster of oncogenes, tumor suppressor genes, cell cycle regulators, cell adhesion molecules and DNA

\section{Biomed Central}


repair genes [4]. However, the precise mechanisms underlying gastric cancer are not well defined.

CDK-associated Cullin1 is a novel gene identified in colorectal carcinoma. It embraces an open reading frame sequence which encodes a $37 \mathrm{kDa}$ protein of 369 amino acids [5]. The CAC1 protein contains a cullin domain between amino acids 137 and 250, and is therefore classified as a member of the cullin family of E3 ubiquitin ligases [5]. Histological investigations had established a possible association of $C A C 1$ expression with pathological features and clinical stages of colorectal carcinoma patients [5]. Moreover, CAC1, in vitro, was expressed in a cell cycledependent manner with high expression in the late G1 to $S$ phase [5]. Notably, CAC1 could promote cell cycle progression and stimulate the kinase activity of $C D K 2$ [5]. Nevertheless, little is known about its expression and biological characteristics in gastric cancer.

The present study was conducted to investigate the expression of $C A C 1$ and to explore the function that $C A C 1$ performs in gastric carcinoma cell lines. The AGS cell line was selected as the model for study because it expressed relatively high level of $C A C 1$, then $C A C 1$ expression was silenced by RNA interference (RNAi), and a series of biological parameters relevant to cell proliferation, cell cycle and apoptosis were examined, correspondingly.

\section{Methods}

\section{Cell culture}

Human gastric cancer cell lines (AGS and MGC803) and gastric mucosa cell line (GES-1) were provided by Central Laboratory of Medical College, Xi'an Jiaotong University, China. Cells were cultured in RPMI 1640 medium (Gibco BRL, Grand Island, NY, USA) supplemented with 10\% newborn calf serum (Gibco BRL, Grand Island, NY, USA), $100 \mathrm{kU} / \mathrm{L}$ penicillin, $0.1 \mathrm{~g} / \mathrm{L}$ streptomycin, $0.3 \mathrm{~g} / \mathrm{L}$ L-glutamine and $0.85 \mathrm{~g} / \mathrm{L} \mathrm{NaHCO}_{3}$ at $37^{\circ} \mathrm{C}$ in a humidified atmosphere containing $5 \% \mathrm{CO}_{2}$.

\section{siRNA transfection}

CAC1-siRNA (sense-GGA UGG UGC CAU AGA UCA ATT 3', antisense-5'UUG AUC UAU GGC ACC AUC CGG3'), CAC1 negative control siRNA (NC-siRNA, sense-5'UUC UCC GAA CGU GUC ACG UTT 3', antisense-5'ACG UGA CAC GUU CGG AGA ATT $3^{\prime}$ ) were chemically synthesized by Shanghai GenePharma Corporation (SGC, Shanghai, China). All siRNAs were mixed into Lipofectamine2000 (Invitrogen, Carlsbad, California, USA) and transfected according to the siRNA Transfection Protocol. The efficiency of CAC1 knockdown was evaluated with qRT-PCR and western blot tests.

\section{MTT assay}

The MTT (3-[4,5-dimethylthiazol-2-yl]-2,5-diphenyltetrazolium bromide thiazolyl blue indicator dye) chemosensitivity assay was applied to determine the proliferation rate of AGS gastric cells. Cells were seeded at a concentration of $5 \times 10^{3}$ cells per well in 96-well plates. All experiments were conducted in triplicate. Cells were incubated for 24 hours and were divided into five groups with five different treatments (null, NC-siRNA (60 nmol/L(nM)), siRNA (30nM), siRNA (60nM), siRNA (90nM)) for 0, 24, 48, 72 and 96 hours, correspondingly. Twenty microliters of $5 \mathrm{mg} / \mathrm{ml}$ MTT (Sigma Chemical Co, St. Louis, $\mathrm{MO}, \mathrm{USA}$ ) in phosphate buffered saline (PBS) were added per well and cells were left inside the incubator for another $4 \mathrm{~h}$ at $37^{\circ} \mathrm{C}$, followed by the addition of $150 \mu \mathrm{l}$ DMSO. Absorbance of the colored solution was measured by a fully automated multi-detection microplate reader (POLARstar OPTIMA, BMG Labtechnologies, Offenburg, Germany) at $490 \mathrm{~nm}$.

\section{Flow cytometric analysis}

Cells $\left(1 \times 10^{5}\right.$ cells/well $)$ were harvested in 6-well plates for 24 hours, and were treated with different agents (null, NC-siRNA, siRNA (60nM)) for 48 hours. Then they were collected to be washed with $0.01 \mathrm{~mol} / \mathrm{L}$ cold $\left(4^{\circ} \mathrm{C}\right)$ PBS by spinning at $800 \mathrm{rpm}, 4^{\circ} \mathrm{C}$ for 8 minutes, and then fixed in $4^{\circ} \mathrm{C}, 75 \%$ ethanol for a night. Fixed cells were centrifuged (as above) and washed again with PBS. Then cells were treated with $100 \mu \mathrm{l}$ of DNase-free, RNaseA $(10 \mathrm{mg} / \mathrm{ml})$ and incubated at $37^{\circ} \mathrm{C}$ for $10 \mathrm{~min}$ utes. Finally, cells were stained with $100 \mu \mathrm{l}$ of $100 \mu \mathrm{g} / \mathrm{ml}$ propidium iodide (light sensitive) and incubated at room temperature for 30 minutes. Cells from every sample were placed in Falcon tubes and read on a FAC sorter (Becton Dickinson, Franklin Lakes, NJ, USA). The cell cycle profiles were interpreted with B-D FAC Sort Cell Quest software.

\section{Apoptosis analysis}

Cells $\left(1 \times 10^{5}\right.$ cells/well $)$ were incubated in 6 -well plates. After 24 hours, they were addressed with different agents (null, cisplatin $(10 \mu \mathrm{M})$, cisplatin $(10 \mu \mathrm{M})+\mathrm{NC}$ siRNA $(60 \mathrm{nM})$, cisplatin $(10 \mu \mathrm{M})+$ siRNA $(60 \mathrm{nM}))$ in serum-free medium for 24 hours, then collected cells were stained with Annexin V/PI using Vybrant apoptosis assay kit No. 2 (Molecular Probes, Eugene, Oregon, USA) and analyzed by flow cytometry.

\section{Quantitative real-time reverse transcription PCR Expression of CAC1 in gastric cancer cell lines}

Total RNA was isolated from various cell lines (AGS, MGC803 and GES-1) using the acid guanidiniumphenol-chloroform method (Trizol, Invitrogen, Carlsbad, USA), and cDNAs were synthesized using the PrimeScriptTM 1st Strand cDNA Synthesis Kit (Invitrogen, Carlsbad, USA). Primer sequences used for amplification 
were designed by TaKaRa (Takara Bio Inc., Shiga, Japan) and listed as follows: forward primer 5'-GCA GCA TAT TCA GAA AGT TCA GA-3'; reverse primer 5'-CAT TTA CAG CCT AAT GCC TTT ACT-3'. $\beta$-Actin forward primer 5'- TGG CAC CCA GCA CAA TGA A -3'; reverse primer 5'- CTA AGT CAT AGT CCG CCT AGA AGC A -3'. Primers were used in regular PCR reactions with cDNA from cells so as to evaluate their proper design and synthesis. Subsequently, the PCR products were sequenced and their similarity to the desired nucleotide sequences confirmed. The relative amount of mRNA was determined using the SYBR GREEN PCR Master Mix (AB, Applied Biosystems, Foster City, California, USA) with gene-specific primers for CAC1 or $\beta$-Actin. All steps were carried out according to the manufacturer's protocol. Real-time PCR reactions were carried out on an ABI 7500 thermal cycler (Applied Biosystems, Foster City, California, USA) with the BioRad iQ5 and MyiQTM Real-time PCR Detection System (BioRad Laboratories, Hercules, California, USA). Three independent PCR tests were performed from each RT sample. The expression of $C A C 1 \mathrm{mRNA}$ in each sample was normalized against $\beta$-actin and the expression level was calculated using the $\Delta \Delta C T$ (delta delta threshold cycle) method.

\section{Expression of apoptosis-related genes in AGS cell line}

RNA isolation and cDNA synthesis were performed as noted. A series of primers were designed and synthesized by TaKaRa including P53 (forward-5'-CCA CCA TCC ACT ACA ACT ACA T-3', reverse-5'-AGG ACA GGC ACA AAC ACG-3'), BCL2 (forward-5'-CAA ATG CTG GAC TGA AAA ATT GTA-3', reverse-5'-TAT TTT CTA AGG ACG GCA TGA TCT-3'), BAX (forward-5'GAC ACC TGA GCT GAC CTT GG-3'; ;everse-5'-AGG AAG TCC AGT GTC CAG C-3') and $\beta$-Actin (forward 5/-TGG CAC CCA GCA CAA TGA A-3'; reverse 5'CTA AGT CAT AGT CCG CCT AGA AGC A -3') genes. As indicated above real-time PCR was performed three times.. The PCR products were detected by measuring the emitting fluorescence at the end of each reaction cycle. The threshold cycle corresponds to the number of cycles required to detect a fluorescence signal above the baseline. The $\beta$-Actin gene served as the internal control of the reaction.

The mRNA expression levels were calculated using the $2^{-\Delta \Delta C T}$ method and expressed in relative quantification units. In detail, the threshold cycles of the housekeeping gene $\beta$-Actin and the target genes BCL2, BAX and P53 were determined in each sample and for each time period. $C T$ values were normalized $(\Delta C T)$ by subtracting the expression levels of the reference gene $\beta$-Actin from the corresponding expression levels of the $B C L 2, B A X$ and $P 53$ in each sample. The normalized gene expression in the treated AGS cells was analyzed relative to their matching untreated cells, which acted as calibrator samples $(\Delta \Delta \mathrm{CT})$.

\section{Western blot examinations}

Fifty micrograms of total protein from the AGS cell line was separated on a $10 \%$ Trisglycine SDS polyacrylamide gel and transferred to a nitrocellulose membrane (BioRad, Hercules, California, USA). The blot was probed with mouse monoclonal antibodies including anti-CAC1 (GeneTex, Irvine, California, USA, 1:1500), anti- $\beta$-Actin (Santa Cruz, CA, USA; 1:5000), anti-P53 (Santa Cruz, CA, USA; 1:2000), anti-BCL2 (Santa Cruz, CA, USA; 1:2000), and anti-BAX (Santa Cruz, CA, USA; 1:2000). Antibody binding was detected using the Gel Blot Imaging Systems (Syngene G:BOX, Cambridge, U.K.) according to the manufacturer's protocol.

\section{Statistical analyses}

All statistical analyses were conducted using SPSS 13.0 software (http://www-01.ibm.com/software/analytics/ spss/). Each assay was performed at least three times. The data were expressed as mean \pm standard deviation, and One-way ANOVA test (two-sided) was performed to determine the significance of differences in multiple comparisons. The results were considered to be statistically significant at $P<0.05$.

\section{Results}

Differential expression of $C A C 1$ in gastric cancer and mucosal cell lines

CAC1 mRNA expression was evaluated with real-time RTPCR in three cell lines. Obviously, CAC1 was expressed in each of the examined cell lines (Figure 1A). However, AGS and MGC803 cell lines showed higher levels of CAC1 mRNA than the GES-1 cell line $(1.0000 \pm 0.0000$ and $0.9507 \pm 0.0176$ versus $0.4340 \pm 0.0414, P<0.05)$. In addition, $C A C 1$ protein expression in western blot examinations showed the same changing trend as CAC1 mRNA (Figure 1A).

\section{Endogenous expression of $C A C 1$ was silenced by the RNAi technique}

Transient transfection of AGS cells with CAC1-siRNA oligos (60nM) designed against CAC1 sequence effectively inhibited the expression of CAC1. CAC1 mRNA expression was examined by real-time RT-PCR. As expected, mRNA level of CAC1 was considerably decreased in CAC1-siRNA group $(1.0000 \pm 0.0000$ versus $0.3583 \pm 0.0244, P<0.05$ ), but showed no significant difference between the control and NC-siRNA groups $(1.0000 \pm 0.0000$ versus $0.9597 \pm 0.0407, P>0.05)$ (Figure 1B). Meanwhile, CAC1 protein expression was also depressed after siRNA treatment in western blot examinations (Figure 1B). 


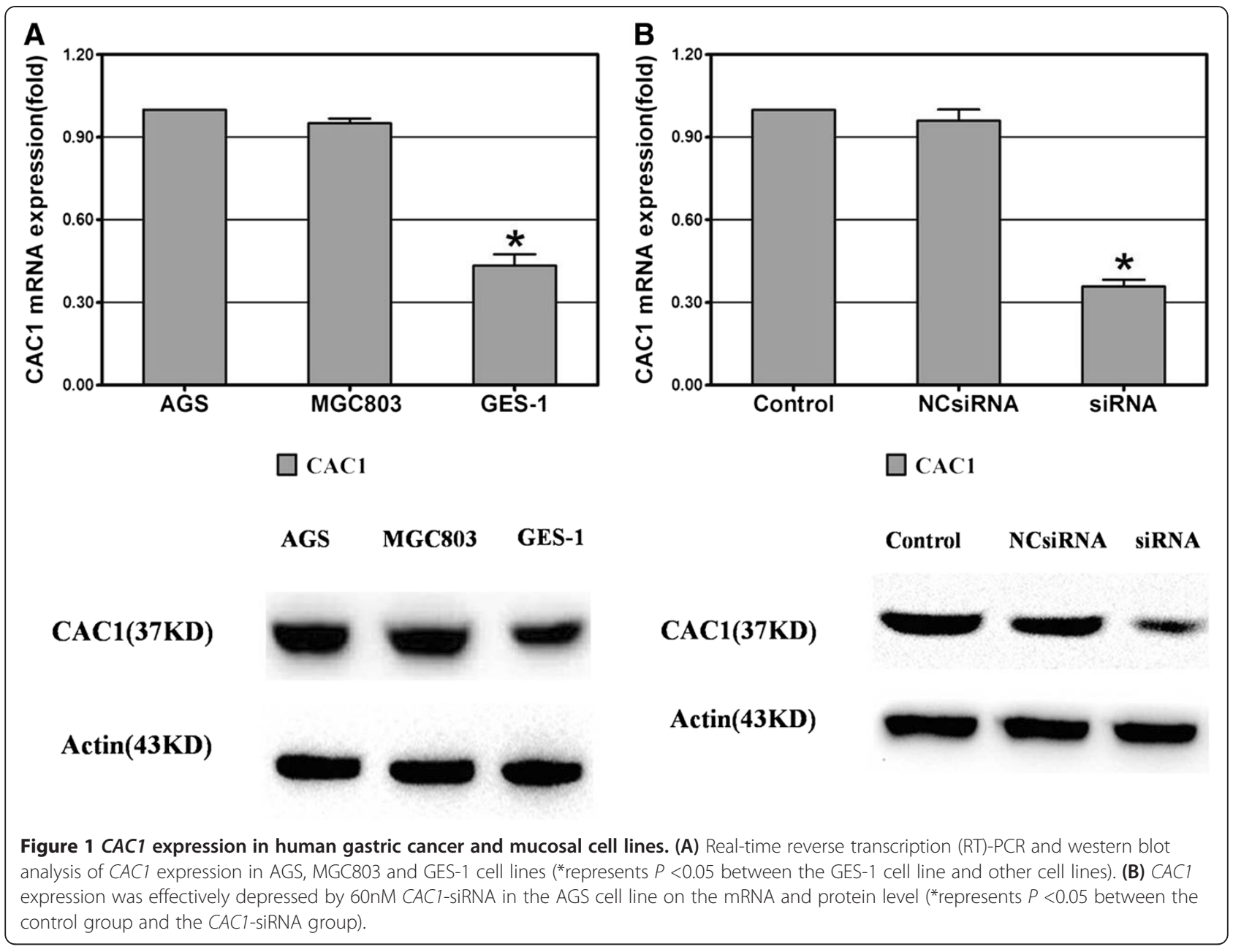

CAC1 silencing inhibits cell proliferation in AGS cell line In order to investigate the role $C A C 1$ plays in the regulation of cell proliferation, AGS cells addressed by transient transfection with CAC1-siRNA of three different dosages (30nM, 60nM and $90 \mathrm{nM}$ ) were subjected to MTT assay. Within four days, CAC1 silencing exhibited a distinct inhibitory effect on cell proliferation according to different doses of CAC1-siRNA (Figure 2). Optical densities (ODs) of the control group, the NC-siRNA group and the 30nM CAC1-siRNA group showed no significant difference with each other $(P>0.05)$, but were higher than those of the $60 \mathrm{nM}$ and 90nM CAC1-siRNA groups $(P<0.05)$. Interestingly, cells treated with $60 \mathrm{nM}$ and 90nM CAC1-siRNA showed almost identical ODs from the beginning to the end $(P>0.05)$.

\section{Cell cycle analysis}

Compared with the control, the proportion of cells treated with CAC1-siRNA increased by $28 \%$ or so in the G1/G0 phase $(45.33 \% \pm 0.82 \%$ versus $73.23 \% \pm 3.04 \%, P<0.05)$, and decreased by approximately $36 \%$ in the $S$ phase $(41.07 \% \pm 1.07 \%$ versus $5.40 \% \pm 5.83 \%, P<0.05)$, with no significant change in the G2/M phase $(13.61 \% \pm 0.46 \%$ versus $21.37 \% \pm 2.88 \%, P>0.05$ ) (Figure 3 ). These results indicate that CAC1 may promote cell cycle progression of AGS cells through the G1/S transition.

Knockdown of CAC1 enhances cisplatin-induced apoptosis The AGS cells were divided into four experimental groups: the control group, the cisplatin-treated $(10 \mu \mathrm{M})$ group, the cisplatin plus NCsiRNA group, and the cisplatin plus CAC1-siRNA (60nM) group. Compared with the control group, the proportions of early/late apoptosis were enhanced with cisplatin treatment, but to a higher extent with cisplatin plus CAC1-siRNA $(2.01 \% \pm$ $0.78 \%$ versus $8.87 \% \pm 3.65 \%$ versus $16.69 \% \pm 3.15 \%$ / $0.57 \% \pm 0.25 \%$ versus $3.89 \% \pm 0.15 \%$ versus $10.01 \% \pm$ $0.09 \%, P<0.05$ ) (Figure $4 \mathrm{~A}$ and $4 \mathrm{~B}$ ), which signified that the knockdown of CAC1 dramatically accelerated cisplatin-induced apoptosis.

\section{Expression profiles of apoptosis-related genes}

The mRNA levels of CAC1, BCL2, BAX and P53 were examined by qRT-PCR. Incubation of the AGS cells with 


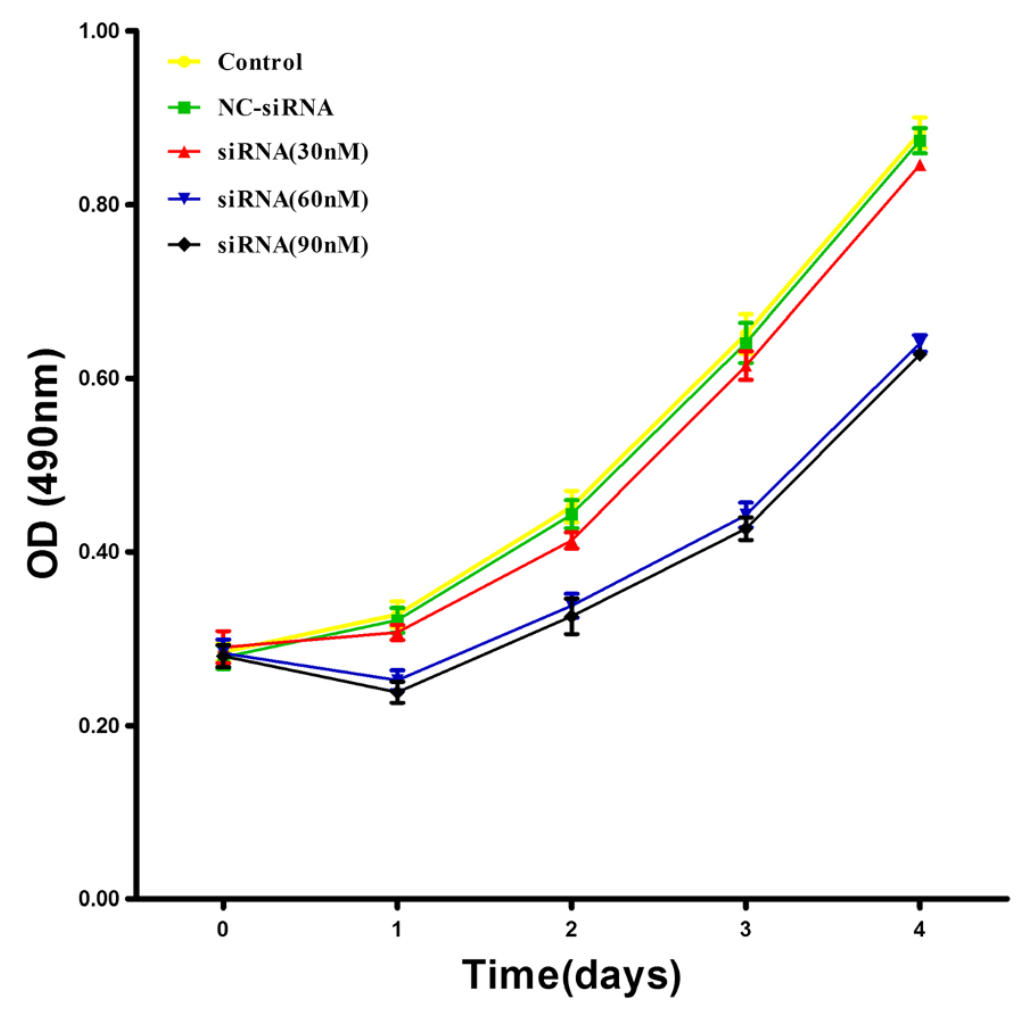

Figure 2 Cell growth was inhibited following CAC1 silencing. AGS cells were transiently transfected with null, NC-siRNA and three concentrations of CAC1-siRNA for four days, respectively. Growth rates of cells were determined by MTT assays.

$10 \mu \mathrm{M}$ cisplatin and/or 60nM CAC1-siRNA for $24 \mathrm{~h}$ did produce interesting mRNA profiles (Figure $5 \mathrm{~A}$ ). Under cisplatin treatment, $C A C 1$ mRNA expression greatly increased, but dropped to a low level when $C A C 1$-siRNA was added simultaneously $(1.0000 \pm 0.0000$ versus $2.1578 \pm 0.2222$ versus $0.3967 \pm 0.0078, P<0.05)$. Compared with the control group, $B C L 2$ mRNA copies showed a $30 \%$ decrease in the cisplatin group, but dropped by around $60 \%$ in the cisplatin plus CAC1siRNA group $(1.0000 \pm 0.0000$ versus $0.7090 \pm 0.0210$ versus $0.4030 \pm 0.0171, P<0.05)$. Conversely, $P 53$ and $B A X$ transcript levels of treated groups were vastly enhanced. Compared with the control group, the P53 expression obtained nearly a two-fold increase in the cisplatin alone group, in addition to a five-fold increase in the cisplatin plus CAC1-siRNA group $(1.0000 \pm 0.0000$ versus $3.0187 \pm 0.1738$ versus $5.9957 \pm 0.3926, P<0.05$ ); the $B A X$ mRNA levels had almost a two- and four-fold augmentation $\quad(1.0000 \pm 0.0000$ versus $3.0237 \pm 0.2581$ versus $4.9897 \pm 0.2923, P<0.05)$, respectively.

Then western blot tests were conducted to detect the protein levels of those genes after CAC1 knockdown. In parallel with the aforementioned mRNA changes, $B A X$ and $P 53$ were markedly upregulated whereas $B C L 2$ was downregulated on the protein level (Figure 5B).

\section{Discussion}

The ubiquitin-proteasome system plays a pivotal role in maintaining the balance between normal growth and uncontrolled proliferation by controlling the abundance of a large variety of cellular proteins [6]. The cullin family of ubiquitin ligases, traditionally composed of CUL1, 2, 3, 4A, 4B, 5 and 7, represents the largest class of RING-type E3 ligases (CRLs) [6]. Without intrinsic catalytic activity, cullins serve as scaffolds that facilitate the assembly of multimeric E3 ligase complexes and transfer ubiquitin from the E2conjugating enzyme to the substrate. Cullin-mediated substrate degradation dictates a wide range of cellular processes such as proliferation, differentiation, and apoptosis. Once the cell regulatory mechanisms of cullin encounter malfunctions or perturbations, accumulation of oncoproteins or excessive degradation of tumor suppressors will inevitably occur, which may provoke cells into malignant transformation and tumorigenesis [6]. In particular, cullin1, the most characterized member of the cullin family, was proven to be closely associated with gastric carcinogenesis, and its overexpression predicts poor prognosis of patients with gastric carcinoma [6,7].

The expression profiles of cancer-related genes in part reflect their biological features in the pathogenesis of 


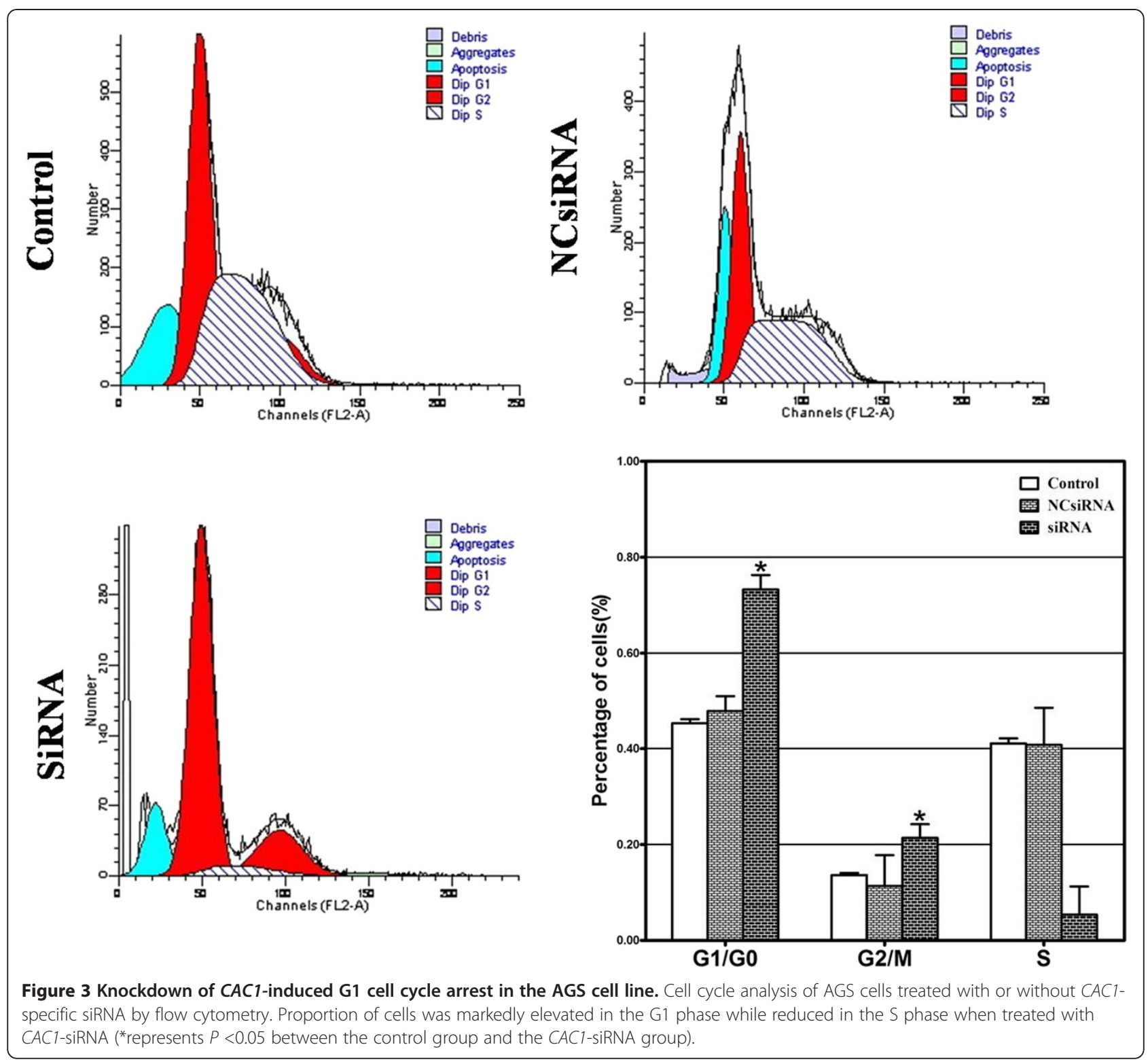

cancer. Being a novel member of the cullin family, CAC1 expression patterns have been extensively investigated in a previous study [5]. Using cDNA library analysis, $C A C 1$ expression was found in normal stomach, small intestine, colon, liver, lung, kidney, muscle, heart, mammary gland, uterus, brain, spleen, lymph node, with high levels in the colon and mammary gland [5]. Western blot tests additionally detected $C A C 1$ protein in a host of normal and cancer cell lines [5]. With regard to our study, the AGS and MGC803 gastric cancer cell lines had higher CAC1 expression than the GES-1 gastric mucosal cell line, which indicate that $C A C 1$ may play an active role in gastric carcinogenesis.

Gene silencing by RNA interference is a powerful method for analyzing gene function [8]. Here, we successfully transfected NCsiRNA and three concentrations of CAC1-siRNA into AGS cells. MTT analyses showed that the proliferation potential of AGS cells was potently inhibited by $60 \mathrm{nM}$ and $90 \mathrm{nM} C A C 1$-siRNA to the same degree, but failed to be influenced by $30 \mathrm{nM}$ CAC1-siRNA or NC-siRNA. It was apparent that CAC1 could positively affect cell proliferation in gastric cancer, which was in accord with previous studies on the HeLa cell line [5]. In MTT examinations, HeLa cells expressing Flag-CAC1 underwent higher proliferation ability than the mock transfected control cells, and cells treated with CAC1-siRNA showed significantly inhibited growth rate [5]. What's more, real-time RT-PCR and western blot analyses confirmed that the expression of CAC1 was efficaciously blocked by $60 \mathrm{nM} C A C 1$-siRNA. Taken 


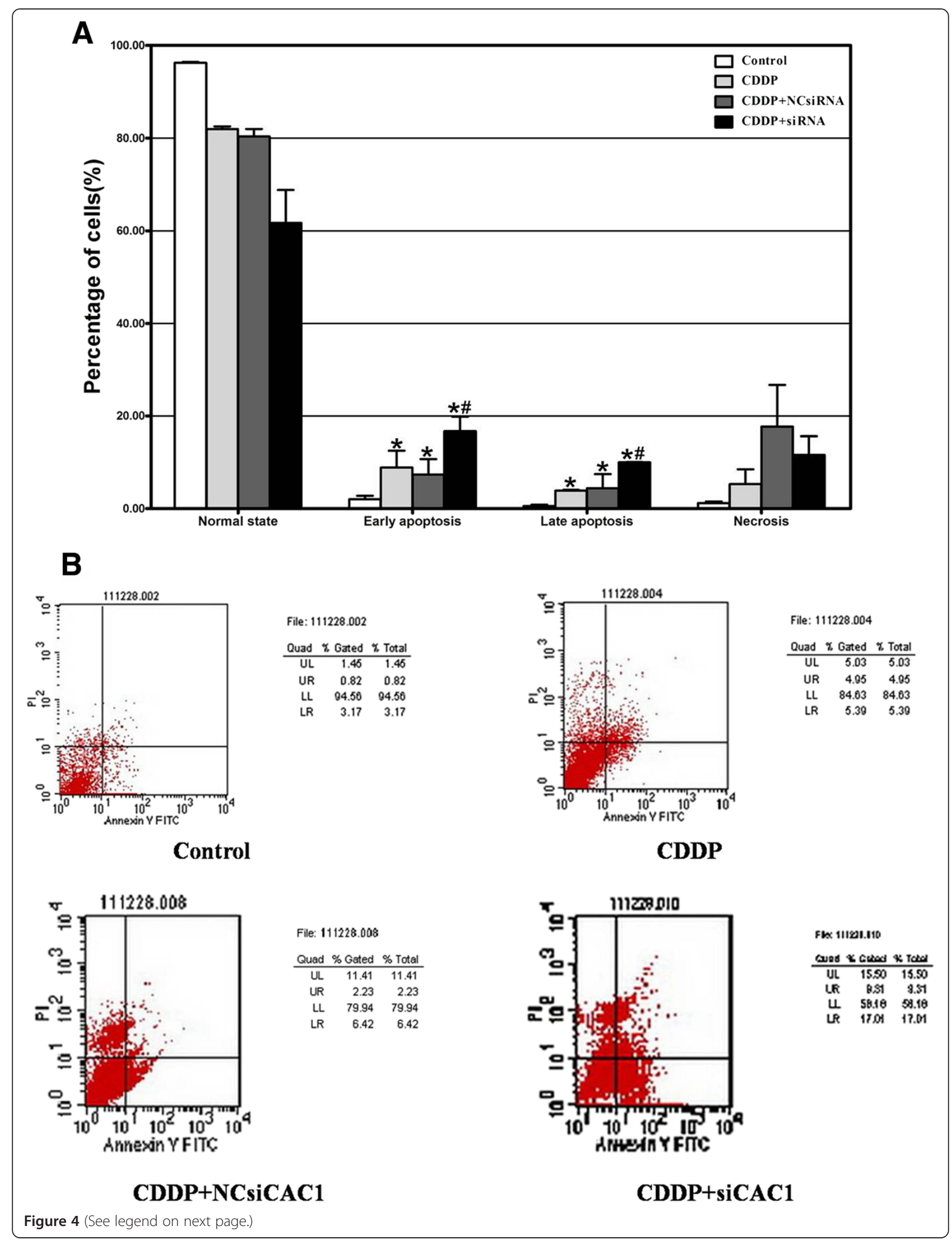


(See figure on previous page.)

Figure 4 CAC1 silencing enhanced cisplatin-induced apoptosis in AGS cell line. (A) The effects of cisplatin alone and in combination with NC-siRNA/CAC1-siRNA on early and late apoptosis of AGS cells ( ${ }^{*}$ represents $P<0.05$ between control group and treated groups; \#represents $P<0.05$ between the cisplatin (CDDP) group and other treated groups). (B) Apoptosis patterns of AGS cells were analyzed by flow cytometry.

together, 60nM was determined to be the most suitable experimental dosage for RNAi in subsequent examinations.

As a general rule, normal cell proliferation depends on orderly and efficient cell cycle process that ensures the duplication and transmission of genetic information from one cell generation to the next. In other words, deregulation of cell proliferation always lies in an abnormal cell cycle. Under $C A C 1$-silencing conditions, flow cytometry in our study revealed elevated proportion of cells in the G1/G0 phase and a reduced rate of cells in the $\mathrm{S}$ phase. In essence, CAC1 knockdown induced G1 cell cycle arrest in the AGS cells, which was consistent with the results from the HeLa cell line [5]. CAC1 was also capable of binding to $C D K 2$ and stimulating its kinase activity at the G1/S phase transition without greatly changing the expressions of cyclinA, cyclinE, cyclinD1, $C D K 2, R B, P T E N$, and so on [5]. It is supposed that $C A C 1$ depression attenuates the activity of $C D K 2$ and interrupts the G1-S transition.

Apoptosis is one of the basic biological phenomena characterized by a series of transformations in cell morphology [9]. Furthermore, apoptosis in concert with cell proliferation forms a crucial balancing mechanism that manages a large number of physiological procedures such as tissue homeostasis and normal development [9]. Under pathological circumstances, aberrant apoptosis usually contributes much to the initiation and progression of cancer [10-12] and even influence the sensitivity of cancer cells to therapeutic interventions [13].

The unequivocal activity of $C A C 1$ in cell cycle regulation raised the possibility that it is, more or less, involved in the process of apoptosis. In the current study, the proportion of early/late apoptotic cells increased with cisplatin treatment, but increased even more so when CAC1 expression was concurrently inhibited by RNAi. That is to say, apoptotic indices of the cisplatin plus CAC1-siRNA group obtained a significant increase in comparison with those of the former three groups with intact $C A C 1$. Cumulative data imply that $C A C 1$ may weaken the anti-cancer effect of cisplatin by counteracting cisplatin-induced apoptosis.

Regulation of apoptosis, however, relies on a network of anti- and proapoptotic molecules such as BCL2 family [14]. BCL2 (B cell CLL/lymphoma 2) is a protooncogene located in the chromosomal region 18q21.3, which codes for an antiapoptotic 26-kDa protein containing four $\mathrm{BH}$ domains (BH1 BH4)) [15]. It can hinder the release of cytochrome $\mathrm{c}$ from the mitochondria, thus abrogating the activation of caspases and finally inhibiting apoptosis [16]. According to early studies, the overexpression of $B C L 2$ drives cells toward malignant transformation [17] and predicts the prognosis in many malignancies [18-22]. Particularly in gastric cancer, frequent expression of the BCL2 gene always occurred in malignant tissues [23-25]. High expression levels of the $B C L 2$ gene, though correlated with less aggression of stomach cancer [26], had a lot to do with drug resistance of the cancer cells [27].

$B A X$ ( $B C L 2$ associated $\mathrm{X}$ protein) gene is located in the human chromosomal region 19q13.3-q13.4 [28]. Its $21-\mathrm{kDa}$ encoding protein, in particular, serves as a proapoptotic member of the $B C L 2$ family. $B A X$ protein consists of three $\mathrm{BH}$ domains (BH1, $\mathrm{BH} 2$, and $\mathrm{BH} 3$ ) and shares a lot of homology with the $B C L 2$ protein. Indeed, $B A X$ protein acts as a suppressor of $B C L 2$ to accelerate apoptotic cell death, by forming $B A X / B C L 2$ complexes or by competing with other $B C L 2$ targets [29]. Overexpression of the $B A X$ gene had a negative effect on cell growth in human gastric cancer, owing to the induction of apoptosis and to the enhancement of cell chemosensitivity [30]. On the contrary, suppression of $B A X$ gene expression induced tumorigenesis in gastric epithelia [23].

Cisplatin is a kind of chemotherapeutic agent widely used in solid malignancies including gastric cancer. It is generally accepted that its primary cytotoxic effect is DNA damage and subsequent induction of apoptosis [31], so variances of apoptosis-associated genes in cisplatin treated cells penetratingly mirror the mechanisms underlying cisplatin-induced apoptosis. As for our study, $C A C 1$ expression was upregulated by cisplatin treatment, but was markedly downregulated by siRNA treatment despite previous cisplatin. Furthermore, underlying the increase of cisplatin-induced apoptosis that follows $C A C 1$ silencing are concomitant gene expression alterations including the upregulation of $P 53$ and $B A X$ as well as the downregulation of $B C L 2$. These effects suggest that $C A C 1$ strengthens cisplatininduced apoptosis by modulating the expression of $B C L 2$, $B A X$ and $P 53$.

As mentioned previously, $B C L 2$ is seemingly situated at the convergence of a couple of apoptotic pathways, and the ratio of $B C L 2$ to $B A X$ protein appears to be the final determinant of whether a cell enters the execution phase [31]. In fact, it is the $B C L 2 / B A X$ ratio that governs the sensitivity of cells to apoptotic stimuli $[32,33]$. In the process of cisplatin-induced apoptosis, $C A C 1$ might protect AGS cells from apoptosis by altering $B C L 2 / B A X$ ratio, for $C A C 1$ silencing brought out a pronounced 
A

CAC1

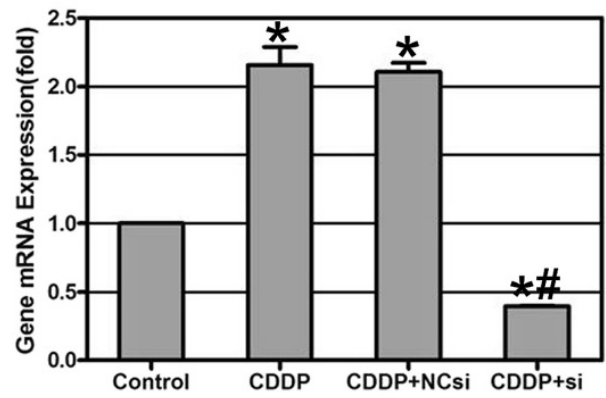

BAX

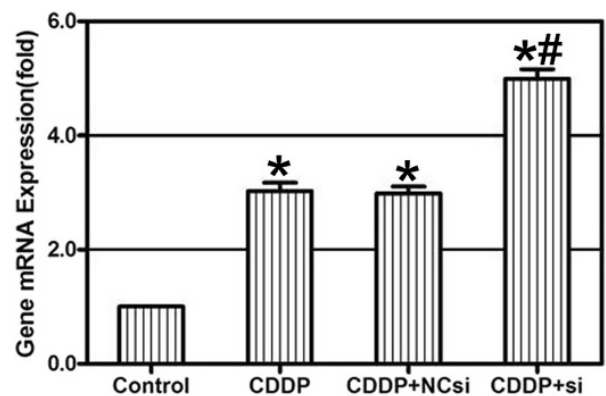

B

CAC1(37KD)

P53(53KD)

BAX(40KD)

BCL2(43KD)

Actin(43KD)

\section{Control CDDP CDDP+NCsi CDDP+si}
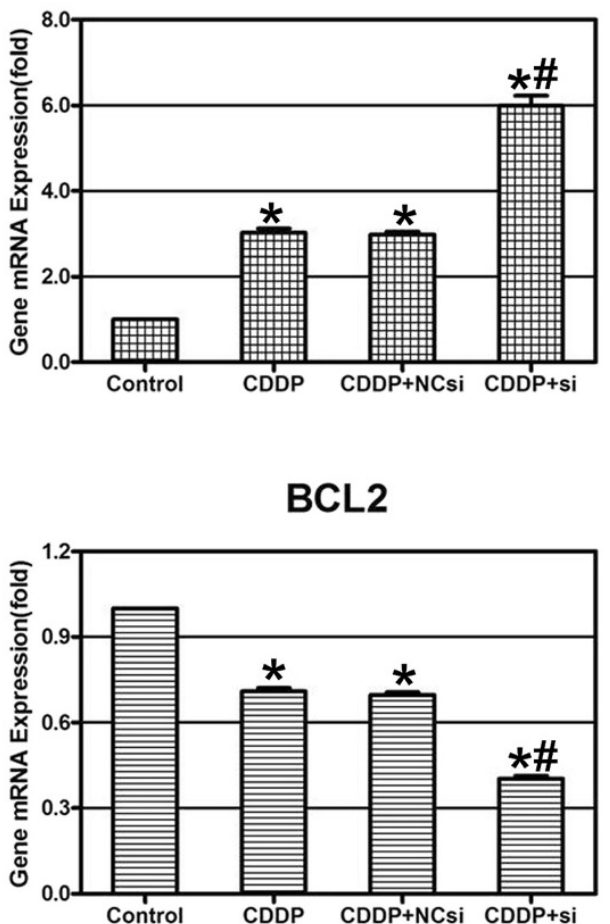

BCL2
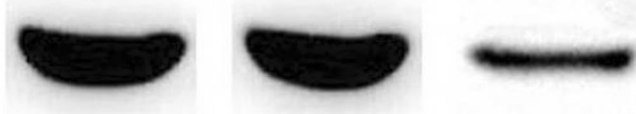
increase of $B A X$ and a decrease of $B C L 2$, which was conducive to the occurrence of cell apoptosis.

Interestingly, the P53 gene in the AGS cells was upregulated with the cisplatin treatment, especially with synchronous suppression of CAC1. It is well known that P53 plays an important role in the management of cell cycle and apoptosis. DNA damage resulting from cisplatin may stimulate expression of the $P 53$ protein that results in both expression of downstream $P 21$ protein and G1 cell cycle arrest [34]. If confronted with irreparable DNA damage, the P53 protein triggers programmed cell death [31]. During cell apoptosis, P53 activates $B A X$ via transcriptional [35] or transcription-independent [36] mechanisms, and represses transcription of BCL2 [14]. Furthermore, P53 can nontranscriptionally induce apoptosis [37]. Therefore, CAC1 inhibition in the AGS cells can bring about excessive $P 53$ accumulation, $B A X$ accumulation, and $B C L 2$ reduction, which ultimately potentiated apoptosis.

The mechanism by which $C A C 1$ functions is not fully clarified. $C A C 1$ was able to reinforce the activity of $C D K 2$ [5], and $C A C 1$ silencing probably impaired the CDK2 activity. An early study argued that CDK2 inhibition could lead to ATM- and ATR-dependent P53 phosphorylation at serine 15 , and thereby cause a significant increase of $P 53$ and $P 21$ protein [38]. With the potential to activate $C D K 2, C A C 1$ is inclined to interfere with the P53-P21 pathway, and thus help AGS cells to resist G1 arrest and apoptosis. On the other hand, CAC1 can serve as a corepressor of $R A R \alpha$ to negatively regulate retinoid acid-induced cellular differentiation and CoRNR box is confirmed to be a major functional region of CAC1 [39]. So it seems that CoRNR box has the potential to regulate cell proliferation and apoptosis, which deserves to be further investigated in other studies.

\section{Conclusion}

In summary, $C A C 1$ exerts multiple biological effects in the AGS gastric carcinoma cell line. Through promoting proliferation and by countering cisplatin-induced apoptosis, $C A C 1$ has been deeply implicated in the pathogenesis and development of gastric cancer. More explorations on specific details of the molecular mechanisms are warranted in the future.

\footnotetext{
Abbreviations

CAC1: CDK-associated Cullin1; CAC1-siRNA: CAC1 small interfering RNA; cisplatin or CDDP: cis-diaminedichloroplatinum; MTT: 3-[4,5-dimethylthiazol2-yl]-2,5-diphenyltetrazolium bromide thiazolyl blue indicator dye; OD: optical density; PBS: phosphate buffered saline; qRT-PCR: quantitative real-time reverse transcription polymerase chain reaction; $\Delta \Delta C T$ : delta delta threshold cycle.
}

\section{Competing interests}

All authors declare that they have no competing interests.

\section{Authors' contributions}

QZ, L-YZ, and K-JN designed the research. QZ, L-YZ, YK, YY, and Z-JL performed the research. QZ and L-YZ analyzed the data. QZ wrote the paper. All authors read and approved the final manuscript.

\section{Author details}

${ }^{1}$ Department of Medical Oncology, First-Affiliated Hospital, Xi'an Jiaotong University, 277 Yanta West Road, Xi'an 710061, Shaanxi Province, China.

${ }^{2}$ Affiliated Shaanxi Provincial Cancer Hospital, College of Medicine, Xi'an Jiaotong University, Xi'an 710061, Shaanxi Province, China. ${ }^{3}$ College of Medicine, Xi'an Jiaotong University, Xi'an 710061, Shaanxi Province, China.

Received: 18 July 2012 Accepted: 23 December 2012

Published: 13 January 2013

\section{References}

1. Jemal A, Bray F, Center MM, Ferlay J, Ward E, Forman D: Global cancer statistics. CA Cancer J Clin 2011, 61:69-90.

2. Parkin D, Bray F, Ferlay J, Pisani P: Global cancer statistics, 2002. CA Cancer J Clin 2005, 55:74-108.

3. Macdonald JS, Smalley SR, Benedetti J, Hundahl SA, Estes NC, Stemmermann GN, Haller DG, Ajani JA, Gunderson LL, Jessup JM, Martenson JA: Chemoradiotherapy after surgery compared with surgery alone for adenocarcinoma of the stomach or gastroesophageal junction. N Engl J Med 2001, 345:725-730.

4. Panani AD: Cytogenetic and molecular aspects of gastric cancer: clinical implications. Cancer Lett 2008, 266:99-115.

5. Kong $Y$, Nan $K$, Yin Y: Identification and characterization of CAC1 as a novel CDK2-associated cullin. Cell Cycle 2009, 8:3544-3553.

6. Lee J, Zhou P: Cullins and cancer. Genes Cancer 2010, 1:690-699.

7. Bai J, Zhou Y, Chen G, Zeng J, Ding J, Tan Y, Zhou J, Li G: Overexpression of Cullin1 is associated with poor prognosis of patients with gastric cancer. Hum Pathol 2011, 42:375-383.

8. Brummelkamp TR, Bernards R, Agami R: A system for stable expression of short interfering RNAs in mammalian cells. Science 2002, 296:550-553.

9. Kerr JF, Wyllie AH, Currie AR: Apoptosis: a basic biological phenomenon with wide-ranging implications in tissue kinetics. Br J Cancer 1972, 26:239-257.

10. Strasser A, Harris AW, Bath ML, Cory S: Novel primitive lymphoid tumours induced in transgenic mice by cooperation between myc and bcl-2. Nature 1990, 348:331-333.

11. Martin SJ, Green DR: Apoptosis and cancer: the failure of controls on cell death and cell survival. Crit Rev Oncol Hematol 1995, 18:137-153.

12. Williams GT: Programmed cell death: apoptosis and oncogenesis. Cell 1991, 65:1097-1098.

13. Green DR, Reed JC: Mitochondria and apoptosis. Science 1998, 281:1309-1312.

14. Thomadaki $\mathrm{H}$, Scorilas A: BCL2 family of apoptosis-related genes: functions and clinical implications in cancer. Crit Rev Clin Lab Sci 2006, 43:1-67.

15. Hanada M, Aimé-Sempé C, Sato T, Reed JC: Structure-function analysis of $\mathrm{BCl}-2$ protein. Identification of conserved domains important for homodimerization with $\mathrm{BCl}-2$ and heterodimerization with Bax. J Biol Chem 1995, 270:11962-11969.

16. Zivny J, Klener P Jr, Pytlik R, Andera L: The role of apoptosis in cancer development and treatment: focusing on the development and treatment of hematologic malignancies. Curr Pharm Des 2010, 16:11-33.

17. McDonnell TJ, Deane N, Platt FM, Nunez G, Jaeger U, McKearn JP, Korsmeyer SJ: bcl-2-immunoglobulin transgenic mice demonstrate extended B cell survival and follicular lymphoproliferation. Cell 1989, 57:79-88.

18. Fontanini G, Vignati S, Bigini D, Mussi A, Lucchi M, Angeletti CA, Basolo F, Bevilacqua $\mathrm{G}$ : $\mathrm{Bcl}-2$ protein: a prognostic factor inversely correlated to p53 in non-small-cell lung cancer. Br J Cancer 1995, 71:1003-1007.

19. Laudanski J, Chyczewski L, Niklińska WE, Kretowska M, Furman M, Sawicki B, Nikliński J: Expression of bcl-2 protein in non-small cell lung cancer: correlation with clinicopathology and patient survival. Neoplasma 1999, 46:25-30.

20. Schorr K, Li M, Krajewski S, Reed JC, Furth PA: Bcl-2 gene family and related proteins in mammary gland involution and breast cancer. J Mammary Gland Biol Neoplasia 1999, 4:153-164. 
21. Karakas T, Maurer U, Weidmann E, Miething CC, Hoelzer D, Bergmann L: High expression of bcl-2 mRNA as a determinant of poor prognosis in acute myeloid leukemia. Ann Oncol 1998, 9:159-165.

22. Ramsay JA, From L, Kahn HJ: bcl-2 protein expression in melanocytic neoplasms of the skin. Mod Pathol 1995, 8:150-154.

23. Konturek PC, Konturek SJ, Sulekova Z, Meixner H, Bielanski W, Starzynska T, Karczewska E, Marlicz K, Stachura J, Hahn EG: Expression of hepatocyte growth factor, transforming growth factor alpha, apoptosis related proteins $\mathrm{Bax}$ and $\mathrm{BCl}-2$, and gastrin in human gastric cancer. Aliment Pharmacol Ther 2001, 15:989-999.

24. Krajewska M, Fenoglio-Preiser CM, Krajewski S, Song K, Macdonald JS, Stemmerman G, Reed JC: Immunohistochemical analysis of Bcl-2 family proteins in adenocarcinomas of the stomach. Am J Pathol 1996, 149:1449-1457.

25. Lauwers GY, Scott GV, Karpeh MS: Immunohistochemical evaluation of bcl-2 protein expression in gastric adenocarcinomas. Cancer 1995, 75:2209-2213.

26. Lee HK, Lee HS, Yang HK, Kim WH, Lee KU, Choe KJ, Kim JP: Prognostic significance of bcl-2 and p53 expression in gastric cancer. Int J Colorectal Dis 2003, 18:518-525.

27. Richardson A, Kaye SB: Pharmacological inhibition of the bcl-2 family of apoptosis regulators as cancer therapy. Curr Mol Pharmacol 2008, 1:244-254.

28. Apte SS, Mattei MG, Olsen BR: Mapping of the human BAX gene to chromosome 19q13.3-q13.4 and isolation of a novel alternatively spliced transcript, BAX delta. Genomics 1995, 26:592-594.

29. Oltvai ZN, Milliman CL, Korsmeyer SJ: BCl-2 heterodimerizes in vivo with a conserved homolog, Bax, that accelerates programmed cell death. Cell 1993, 74:609-619.

30. Komatsu K, Suzuki S, Shimosegawa T, Miyazaki II, Toyota T: Cre-loxPmediated bax gene activation reduces growth rate and increases sensitivity to chemotherapeutic agents in human gastric cancer cells. Cancer Gene Ther 2000, 7:885-892.

31. Fisher DE: Apoptosis in cancer therapy: crossing the threshold. Cell 1994 78:539-542.

32. Gazzaniga P, Gradilone A, Vercillo R, Gandini O, Silvestri I, Napolitano M, Albonici L, Vincenzoni A, Gallucci M, Frati L, Agliano AM: Bcl-2/bax mRNA expression ratio as prognostic factor in low-grade urinary bladder cancer. Int J Cancer 1996, 69:100-104.

33. Thomas A, El Rouby S, Reed JC, Krajewski S, Silber R, Potmesil M, Newcomb EW: Drug-induced apoptosis in B-cell chronic lymphocytic leukemia: relationship between $\mathrm{p} 53$ gene mutation and bcl-2/bax proteins in drug resistance. Oncogene 1996, 12:1055-1062.

34. Reed E: Cisplatin. Cancer Chemother Biol Response Modif 1999, 18:144-151.

35. Vousden KH, Lu X: Live or let die: the cell's response to p53. Nat Rev Cancer 2002, 2:594-604.

36. Chipuk JE, Kuwana T, Bouchier-Hayes L, Droin NM, Newmeyer DD, Schuler M, Green DR: Direct activation of Bax by p53 mediates mitochondrial membrane permeabilization and apoptosis. Science 2004, 303:1010-1014.

37. Fridman JS, Lowe SW: Control of apoptosis by p53. Oncogene 2003, 22:9030-9040

38. Zhu Y, Alvarez C, Doll R, Kurata H, Schebye XM, Parry D, Lees E: Intra-S-phase checkpoint activation by direct CDK2 inhibition. Mol Cell Biol 2004, 24:6268-6277.

39. Moon M, Um SJ, Kim EJ: CAC1 negatively regulates RARa activity through cooperation with HDAC. Biochem Biophys Res Commun 2012, 427:41-46.

\section{Submit your next manuscript to BioMed Central and take full advantage of:}

- Convenient online submission

- Thorough peer review

- No space constraints or color figure charges

- Immediate publication on acceptance

- Inclusion in PubMed, CAS, Scopus and Google Scholar

- Research which is freely available for redistribution

Submit your manuscript at www.biomedcentral.com/submit 